\title{
Higher Ion Selectivity with Lower Energy Usage Promoted by Electroosmotic Flow in the Transport through Conical Nanopores.
}

\author{
Warren Brown, Maksim Kvetny, Ruoyu Yang, Gangli Wang* \\ Department of Chemistry, Georgia State University, Atlanta, GA 30302
}

Table of Contents:

Simulation Details

Scheme S 1: Simulation Structure

Table S 1: Bounday conditions for Nernst-Planck, Navier-Stokes and Poisson equations.

\section{Further descriptions}

Figure S 1: . Radial distributions of the $\mathrm{K}^{+}$flux (z component, through nanopore) at $\mathrm{z}$ cutlines 10,100 and 1000 $\mathrm{nm}$. The radius of the nanopore was $20 \mathrm{~nm}$.

Figure S 2: Radial distributions of the $\mathrm{Cl}^{-}$flux (z component, through nanopore) at z cutlines 10, 100 and 1000 $\mathrm{nm}$. The radius of the nanopore was $20 \mathrm{~nm}$.

Scheme S 2. Fluid velocity distributions in the two reservoirs of a $20 \mathrm{~nm}$-nanopore in $1 \mathrm{mM} \mathrm{KCl}$ solution at $\sim 0.7$ V.

Figure S 3: Cation selectivity $\left(\mathrm{t}_{+}\right)$in PNP, PNP-NS results over time under the time-dependent applied potential waveform.at $\mathrm{HC}$ for a $20 \mathrm{~nm}$ radius nanopore.

Figure S 4: Transport at varied potential scan rates simulated with PNP model without EOF consideration.for overall current $\mathrm{i}_{\text {Total }}$, cation $\mathrm{i}_{\mathrm{K}}{ }^{+}$, anion $\mathrm{i}_{\mathrm{Cl}}{ }^{-}$and $\mathrm{t}+$ in a 40 -nm radius nanopipette.

Figure S 5: Overall dissipated energy and forward and backward components for PNP and PNP-NS for a $20 \mathrm{~nm}$ nanopore.

Figure $\mathrm{S}$ 6: Energy dissipation $\mathrm{E}_{\mathrm{diss}}$ at the low conductivity states with a $20 \mathrm{~nm}$ radius nanopore at different ionic strength.

Figure S 7: The simulated I-V curves with PNP and PNP-NS using a $20 \mathrm{~nm}$ nanopore.

Figure S 8: Individual ion conductivity with PNP and PNPNS models corresponding to the curves in Figure S 7. Figure S 9: The simulated I-V curves ( $i_{\text {total }}$ ) with PNP-NS corresponding to the data in Figure 6 with a $40 \mathrm{~nm}$ and $100 \mathrm{~nm}$ radius nanopore.

Figure S10: The $t_{+}$from PNP-NS at additional concentrations (Figure 6). The nanopore radii are $20 \mathrm{~nm}, 40 \mathrm{~nm}$ and $100 \mathrm{~nm}$.

Figure S 11: The energy dissipation at LC and cation selectivity ( $\mathrm{t}+$ at $-1 \mathrm{~V})$ of different sized nanopores in different ion concentrations.

Figure S 12: The energy dissipation and cation selectivity from PNP model at HC and LC from a $20 \mathrm{~nm}$ nanopore. 


\section{Simulation Details}

The simulations were carried out using COMSOL multiphysics version 4.3 (and 5.5 due to the update not the function/outcome) using 'Electrostatics' (ES) for the Poisson equation; 'Transport of Diluted Species' (TDS) for the Nernst-Planck equation; and 'Laminar Flow' (LF) for the Navier-Stokes equation. In scheme S1: 1-2 (5 $\mu \mathrm{m})$ is the reference electrode; 2-3 $(2 \mu \mathrm{m})$ is the length of the reservoir near to the reference electrode; 4-7 encompass the surface surface charge density (SCD);8-9 $(2 \mu \mathrm{m})$ is the length of the reservoir near the working electrode $3 ; 9-10(5 \mu \mathrm{m})$ is the working electrode; 3-4 and 7-8 are uncharged areas of the nanopore; and 3-8 (10 $\mu \mathrm{m})$ is the length of the pore . rt is the radius of the nanopore (20, 40 and $100 \mathrm{~nm})$. Boundaries 5-6 and 6-7 have a gradient surface charge density that decays from a maximum of $70 \mathrm{mC} / \mathrm{m} 2$ at the tip opening to $1 \mathrm{mC} / \mathrm{m} 2$ as the bulk/flat surface as previously reported.1 Half of the nanopore is defined for centerline (1-10) integrations to obtain current/flux. The current is obtained by radial intergration at the cutline $\mathrm{z}=100 \mathrm{~nm}$ (100 nm into the nanopore from the small opening towards larger opening).

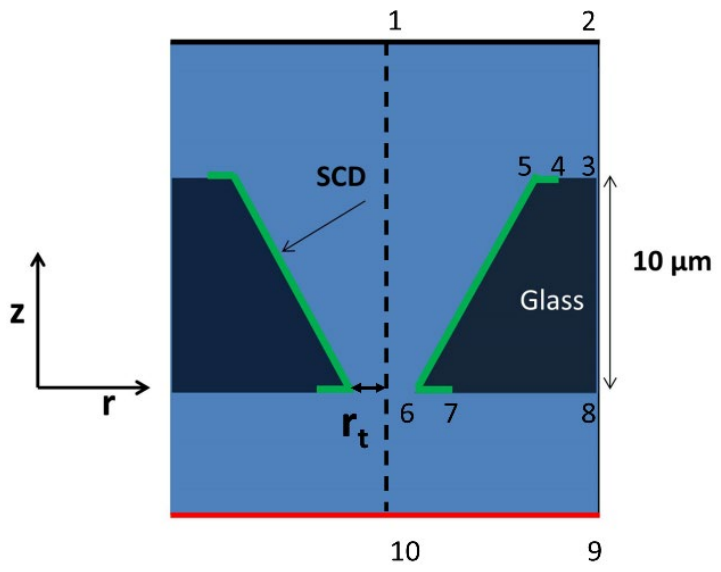

Scheme S 1. Simulation structure.

The Nernst-Planck equation is given by:

$\boldsymbol{J i}=-D i \nabla c i+\frac{z i F}{R T} D i \operatorname{ci} \nabla \phi+c i \mathbf{u}$

For the ion $i \boldsymbol{J i}$ is the flux; $z i$ is the charge; $R$ is the gas constant; $F$ is Faraday's constant; $D i$ is the diffusion coefficient; $c i$ is the concentration; $\phi$ is the potential; $\mathbf{u}$ is the fluid velocity; $T$ is the temperature. For the PNP model the third term is neglected and for the PNP-NS model the term is the EOF. 'TDS' in table S1 represents the boundary conditions for the Nernst-Planck equation.

The Poisson equation is given by:

$\nabla^{2}\left(\varepsilon_{0} \varepsilon_{r} \phi\right)=-F \sum z i c i$

$\varepsilon_{0}$ is the relative permittivity of free space and $\varepsilon_{r}$ is the relative permittivity of the medium in this case water. 'ES' in table $\mathrm{S} 1$ is the boundary conditions for the Poisson equation.

The Navier-Stokes equation is given by:

$\frac{1}{\rho}\left(-\nabla p+\eta \nabla^{2} \mathbf{u}-\left(F \sum z i c i\right) \nabla \phi\right)=0$

$\rho$ is the density, $p$ is the pressure and $\eta$ is the viscosity. 'LF' in table S1represents the boundary conditions for the NavierStokes equation. 


\begin{tabular}{|l|l|l|l|l|l|l|l|l|l|}
\hline $\begin{array}{l}\text { COMSOL } \\
\text { Modules }\end{array}$ & $1-2$ & $2-3$ & $3-4$ & $4-5$ & $5-6$ & $6-7$ & $7-8$ & $8-9$ & $9-10$ \\
\hline TDS & Conc =bulk & No flux & No flux & No flux & No flux & No flux & No flux & No flux & Conc =bulk \\
\hline ES & Ground & SCD $=0$ & SCD $=0$ & $\begin{array}{l}\text { SCD }= \\
-1 \mathrm{mC} \mathrm{m}^{-2}\end{array}$ & $\begin{array}{l}\text { SCD }=\text { Exponential } \\
\text { decay (Gradient) }\end{array}$ & $\begin{array}{l}\text { SCD }=\text { Exponential } \\
\text { decay (Gradient) }\end{array}$ & SCD $=0$ & SCD =0 & Working \\
\hline LF & Outlet $(\mathrm{p}=0)$ & No slip & No slip & No slip & No slip & No slip & No slip & No slip & Inlet (p=0) \\
\hline
\end{tabular}

Table S 1. Boundary Conditions

Note that the surface charge density on surfaces between 5-7 are defined by an exponential gradient. See our previous report for justification and details on these boundary conditions. ${ }^{1}$

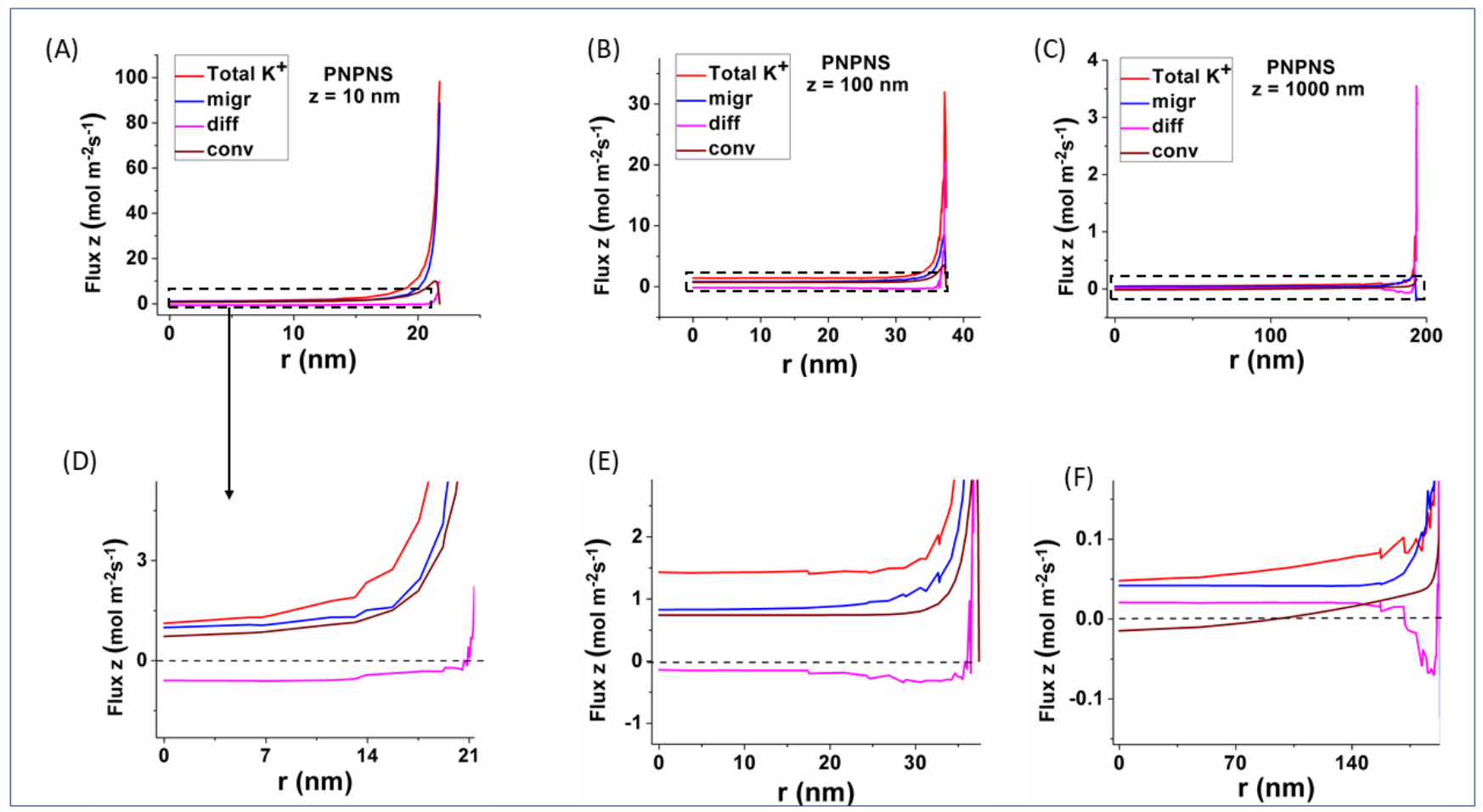

Figure S 1. Radial distributions of the $\mathrm{K}+$ flux (z component, through nanopore) at $10 \mathrm{~nm}(\mathrm{~A}), 100 \mathrm{~nm}(\mathrm{~B})$ and $1000 \mathrm{~nm}$ (C). Zoom in views of the top panels (dashed rectangle) are shown directly below in panels (D), (E) and (F). The dashed line at zero flux indicates the direction of the flux. Positive flux means ions are transported from the small opening to the larger opening and vice versa. The radius of the nanopore was $20 \mathrm{~nm}$ at a scan rate $100 \mathrm{~V} / \mathrm{s}$. Decrease in mesh sized will further improve the smoothness of the flux profiles near the wall but not affect the integrated current or transport analysis, at the cost of longer simulation time. 

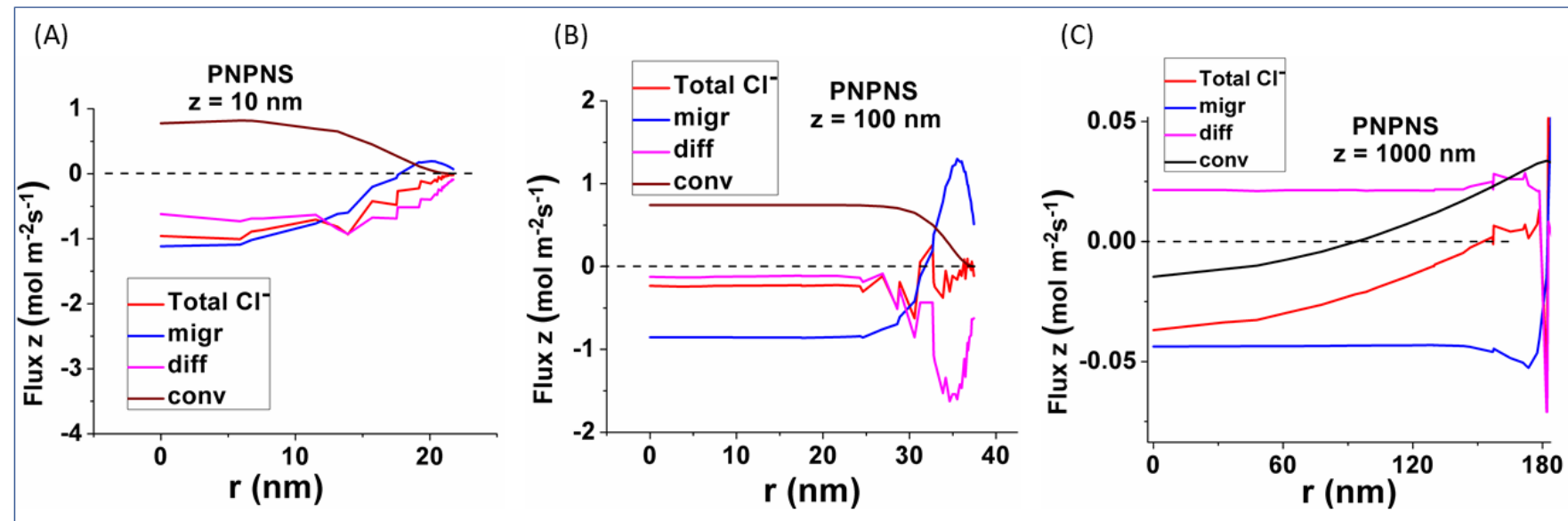

Figure S 2. Radial distributions of the Cl- flux (z component, through nanopore) at $10 \mathrm{~nm}(\mathrm{~A}), 100 \mathrm{~nm}$ (B) and $1000 \mathrm{~nm}$ (C). The dashed line at zero flux indicates the direction of the flux. Positive flux means ions are transported from the small opening to the larger opening and vice versa. The radius of the nanopore simulation was $20 \mathrm{~nm}$ at a scan rate $100 \mathrm{~V} / \mathrm{s}$.

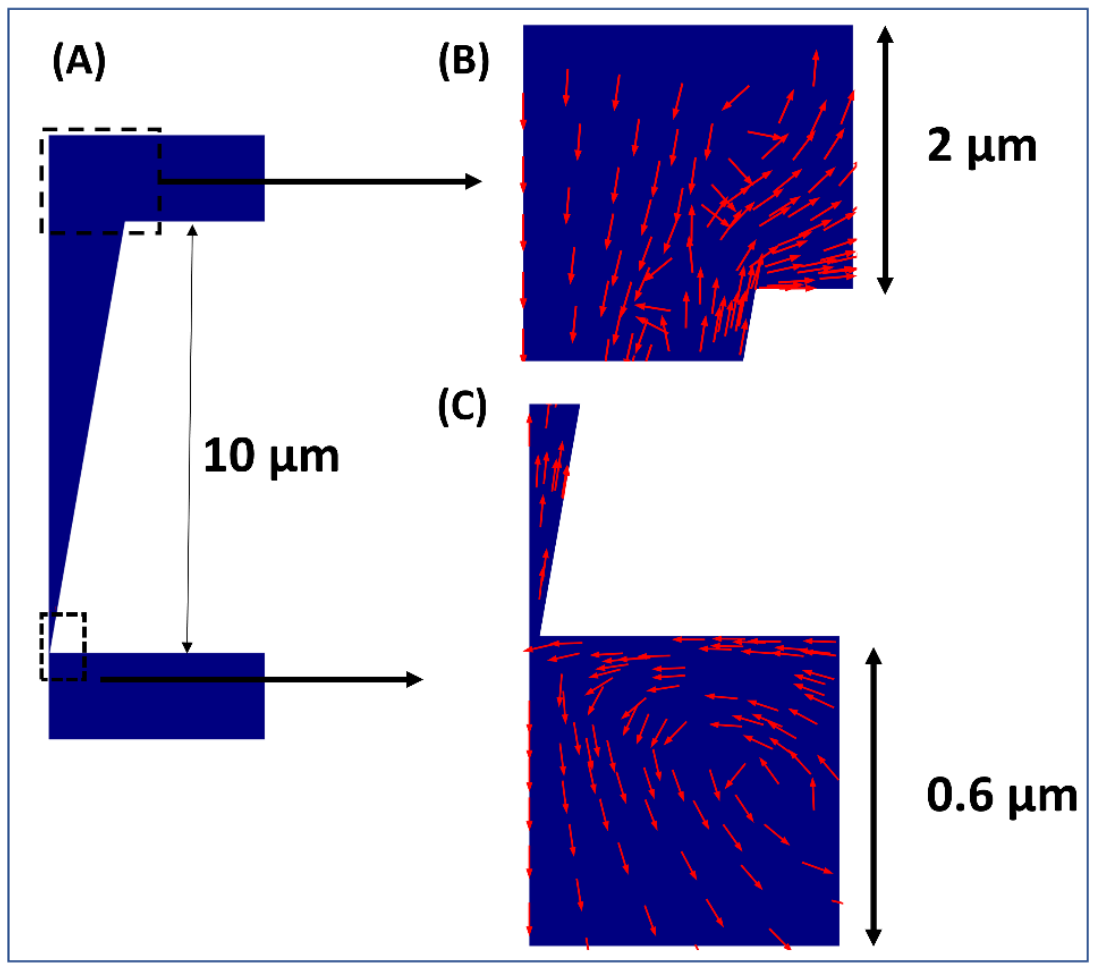

Scheme S 2. Fluid velocity distributions in the two reservoirs of a $20 \mathrm{~nm}$-nanopore in $1 \mathrm{mM} \mathrm{KCl}$ solution at $\sim 0.7 \mathrm{~V}$. Overall simulation structure (A), reservoir velocity distributions outside the large (B) and small opening (C). Dashed boxes indicate the approximate area of the structure that is enlarged in panels $\mathrm{B}$ and $\mathrm{C}$. 


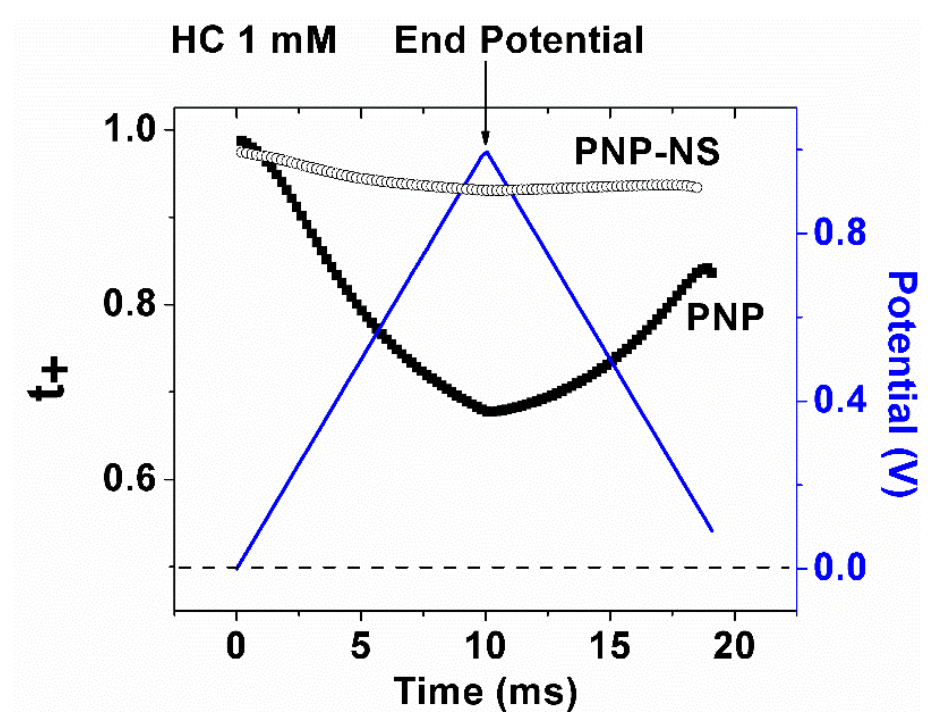

Figure S 3. Cation selectivity ( $\mathrm{t}+$ ) in PNP (solid), PNP-NS (Open) results over time under the time-dependent applied potential waveform. Data from Figure 2D, a $20 \mathrm{~nm}$ radius nanopore at $\mathrm{HC}$ states in $1 \mathrm{mM} \mathrm{KCl}$. 


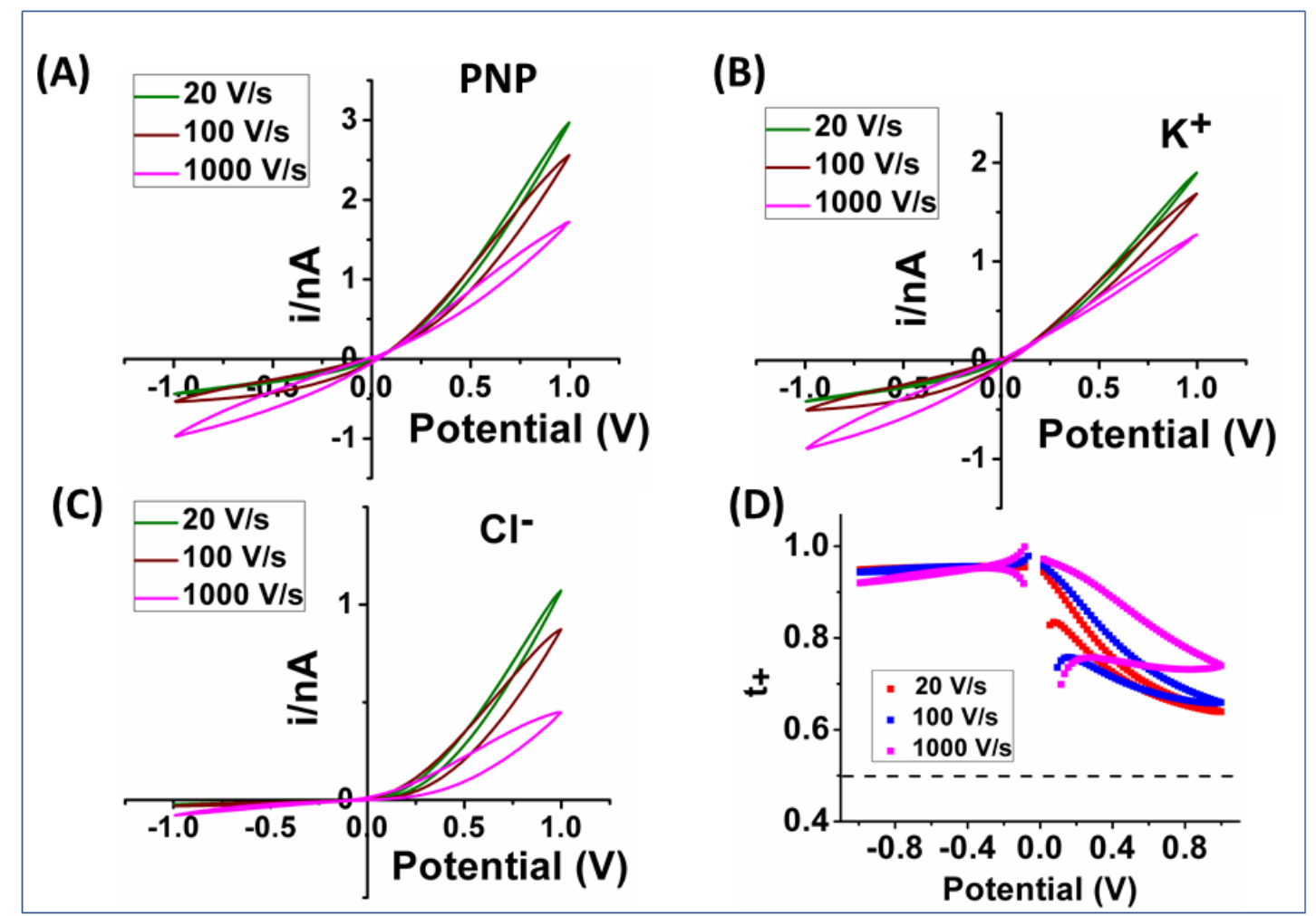

Figure S 4. Transport at varied potential scan rates simulated with PNP model without EOF consideration. The overall current iTotal (A), cation $\mathrm{iK}+(\mathrm{B})$, anion $\mathrm{iCl}-(\mathrm{C})$ and $\mathrm{t}+(\mathrm{D})$ were obtained using a 40-nm nanopipette in $1 \mathrm{mM} \mathrm{KCl}$ solution. Note the $\mathrm{iCl}-$ in $\mathrm{C}$ is ten times lower in y scale.

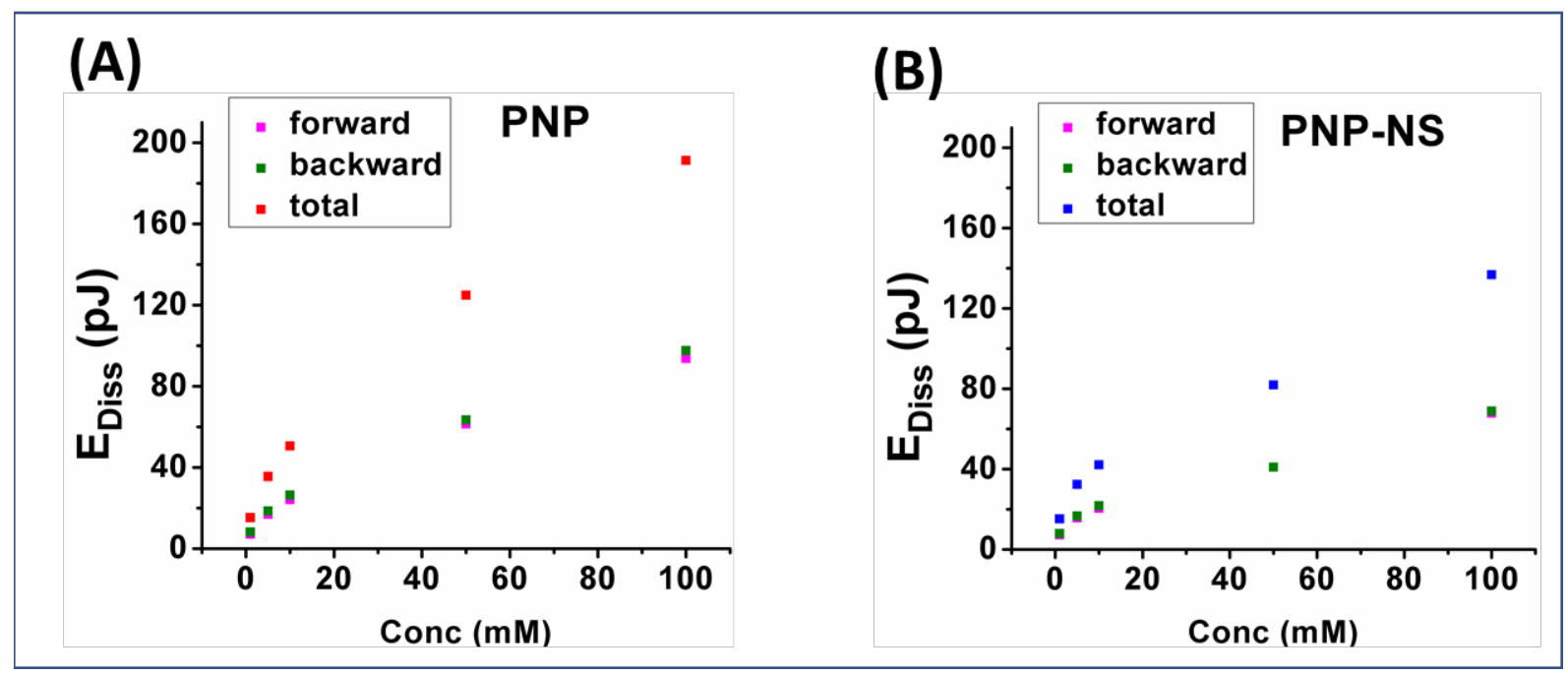

Figure S 5. Overall dissipated energy and forward and backward components from (A) PNP and (B) PNP-NS models with a $20 \mathrm{~nm}$ nanopore. Data calculated from the $\mathrm{i}-\mathrm{V}$ loops at the high conductivity states. 


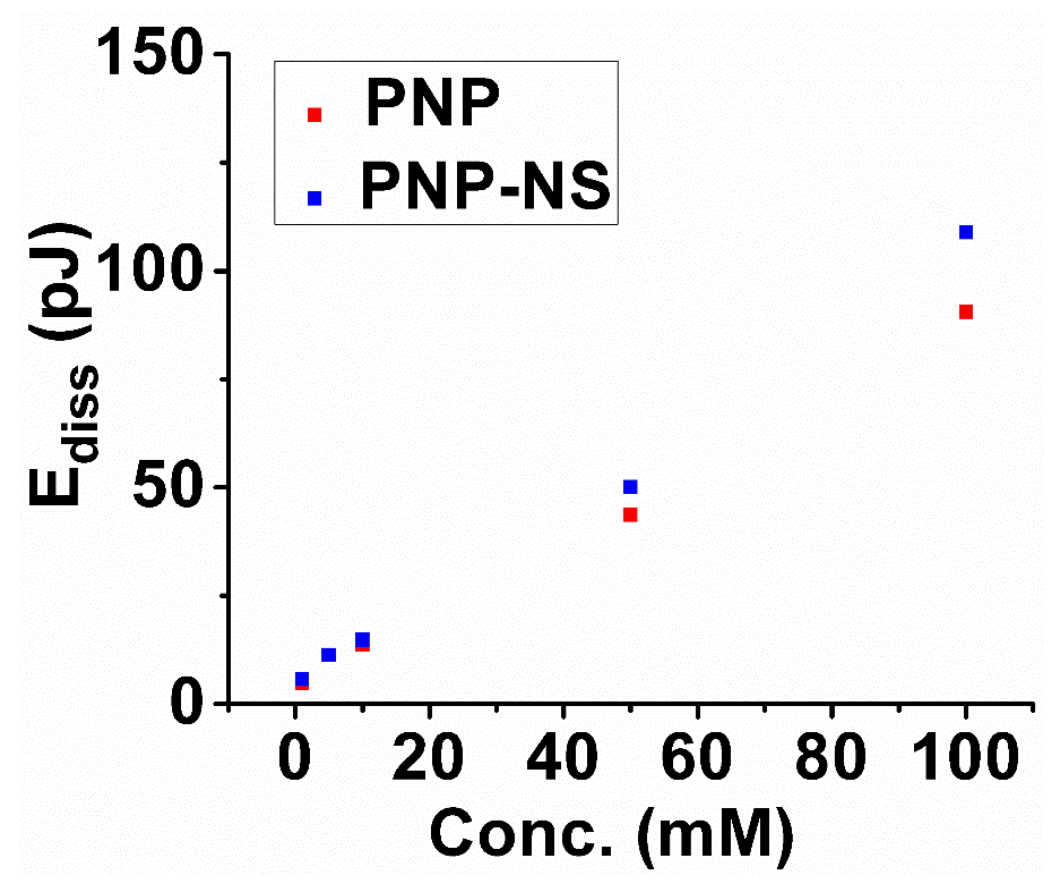

Figure S 6. Energy dissipation Ediss at the low conductivity states with a $20 \mathrm{~nm}$ radius nanopore at different ionic strength.

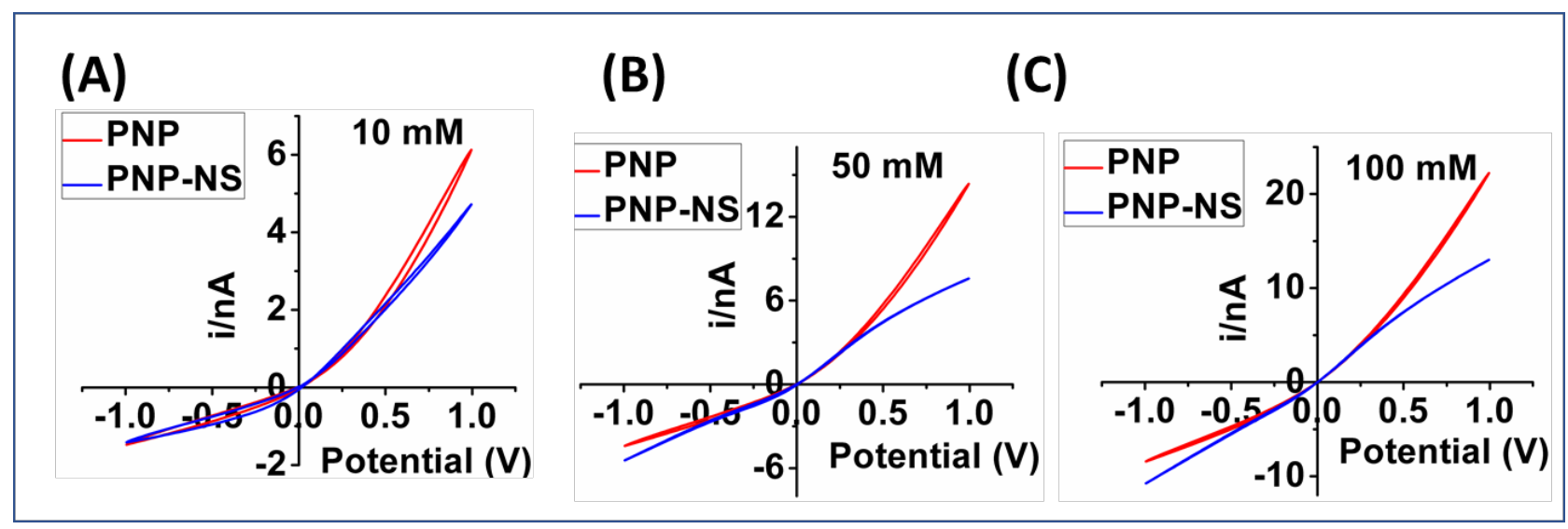

Figure S 7. The simulated I-V curves with PNP (red) and PNP-NS (blue) using a $20 \mathrm{~nm}$ nanopore corresponding to the data in Figure 6. 


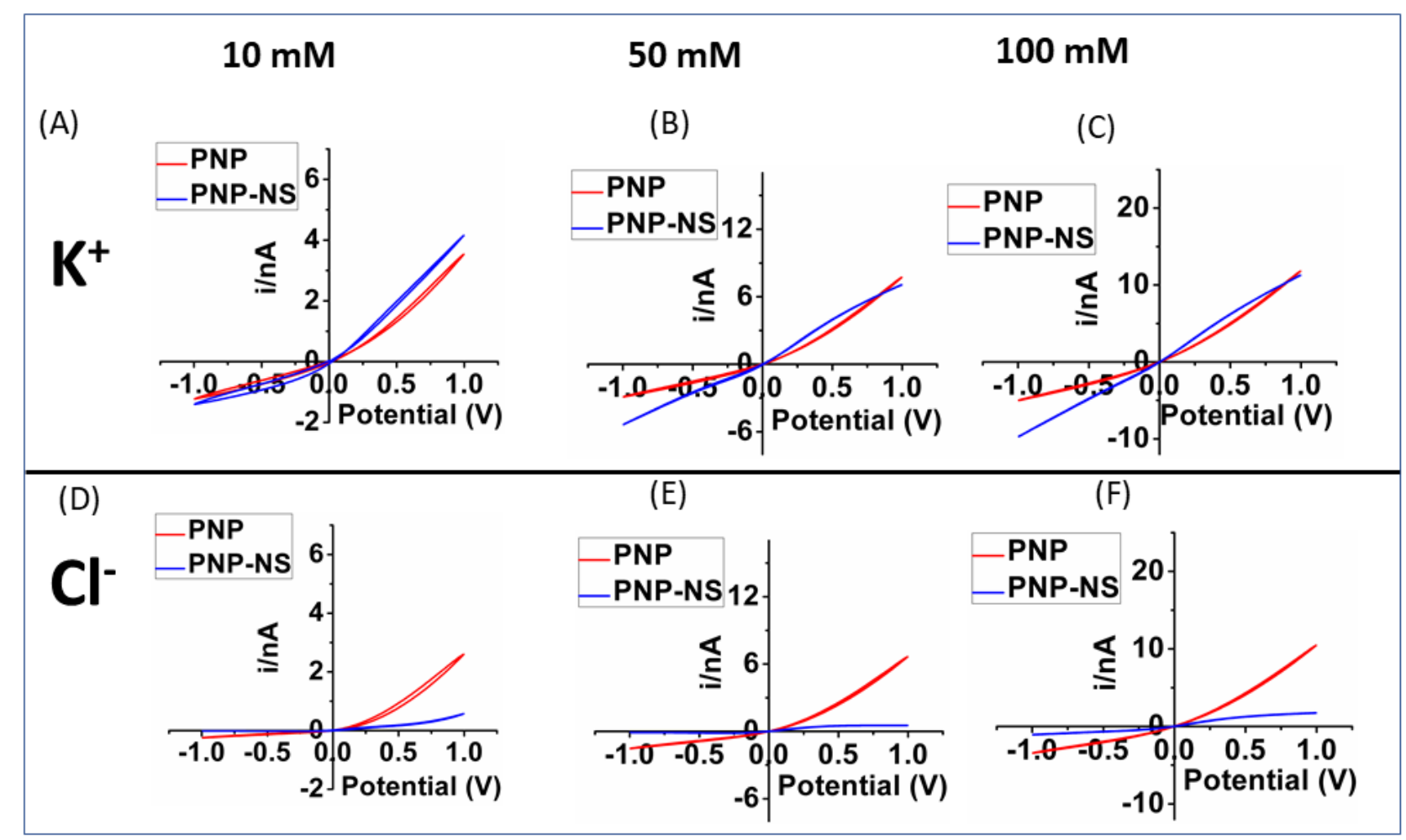

Figure S 8. Individual ion conductivity with PNP (red) and PNPNS (blue) models corresponding to the curves in Figure S 9. $\mathrm{K}+$ measurements at $10 \mathrm{mM}(\mathrm{A}), 50 \mathrm{mM}$ (B) and $100 \mathrm{mM}(\mathrm{C})$. Corresponding Cl- measurements for each concentration directly below in panels (D), (E) and (F). The radius of the nanopore simulation was $20 \mathrm{~nm}$ and scan rate $100 \mathrm{~V} / \mathrm{s}$. 


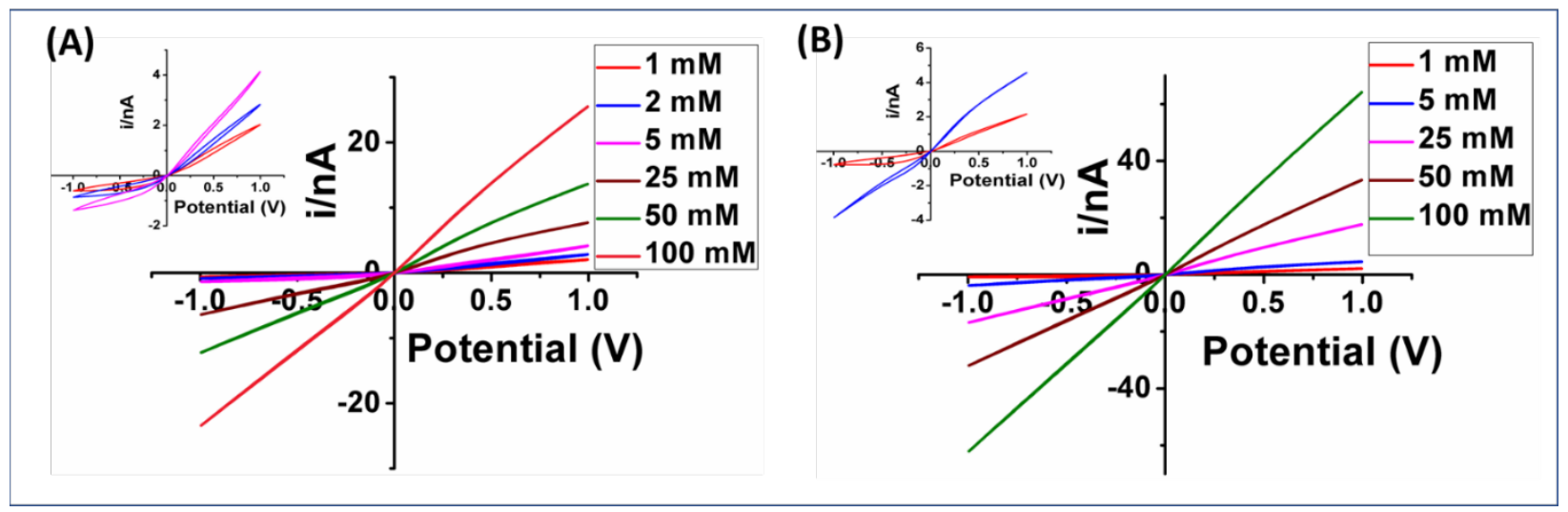

Figure S 9. The simulated I-V curves (itotal) with PNP-NS corresponding to the data in Figure 6 with a (A) $40 \mathrm{~nm}$ and (B) $100 \mathrm{~nm}$ radius nanopore. Insets show lower concentrations for easier viewing. Scan rate at $100 \mathrm{~V} / \mathrm{s}$.

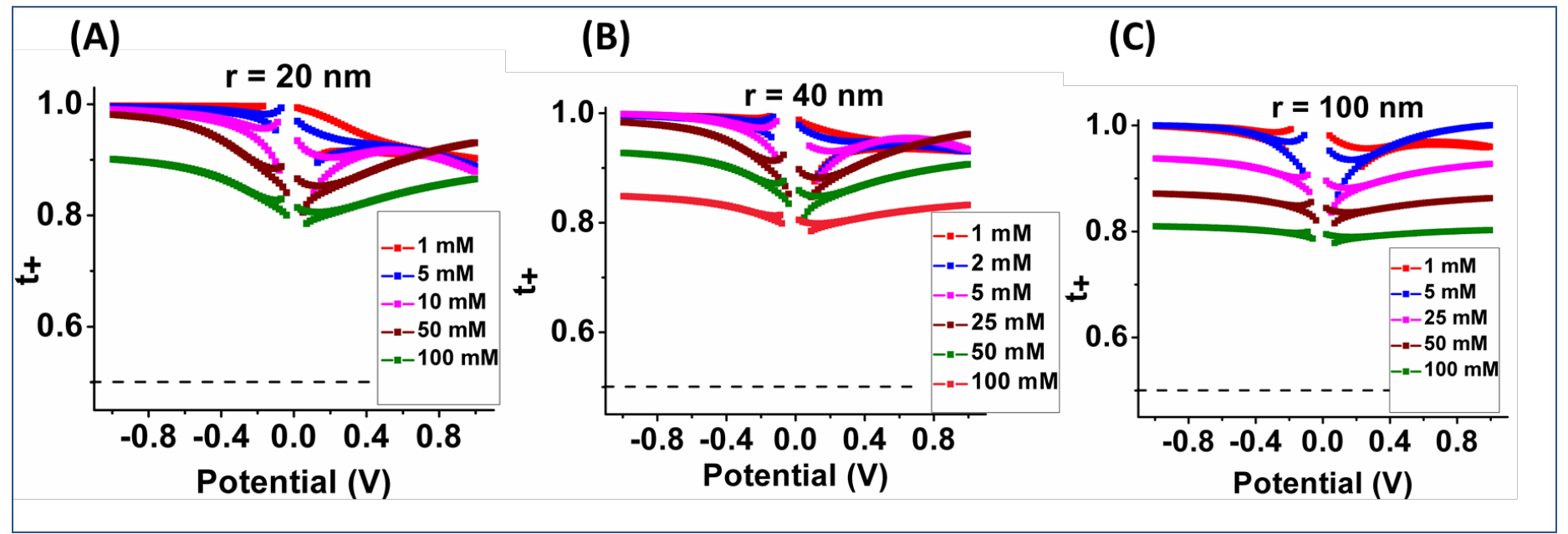

Figure S 10. The $\mathrm{t}+$ from PNP-NS at additional concentrations (Figure 6). The nanopore radii are (A) $20 \mathrm{~nm}$, (B) $40 \mathrm{~nm}$ and (C) $100 \mathrm{~nm}$. Scan rate at $100 \mathrm{~V} / \mathrm{s}$. 


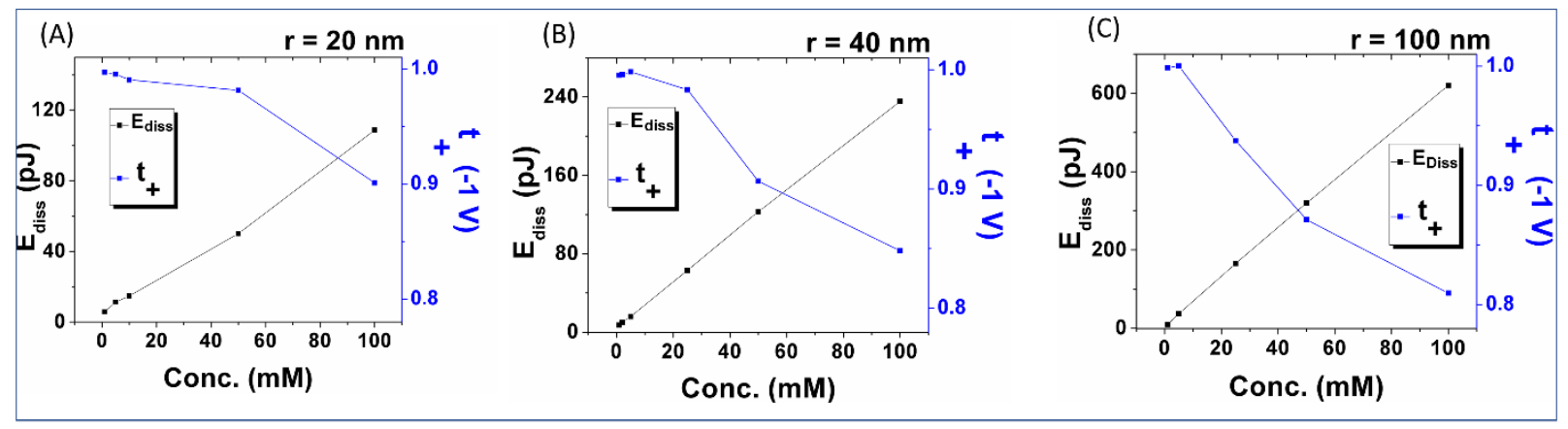

Figure S 11. The energy dissipation at LC and cation selectivity $(\mathrm{t}+$ at $-1 \mathrm{~V})$ of different sized nanopores in different ion concentrations. (A), (B) and (C) are simulated with $20 \mathrm{~nm}, 40 \mathrm{~nm}$ and $100 \mathrm{~nm}$ radius nanopipette with PNP-NS. Note Ediss is an integrated value over the time of $\mathrm{LC}$ loop while $\mathrm{t}+$ at $-1 \mathrm{~V}$ was used as a representative point.

(A)

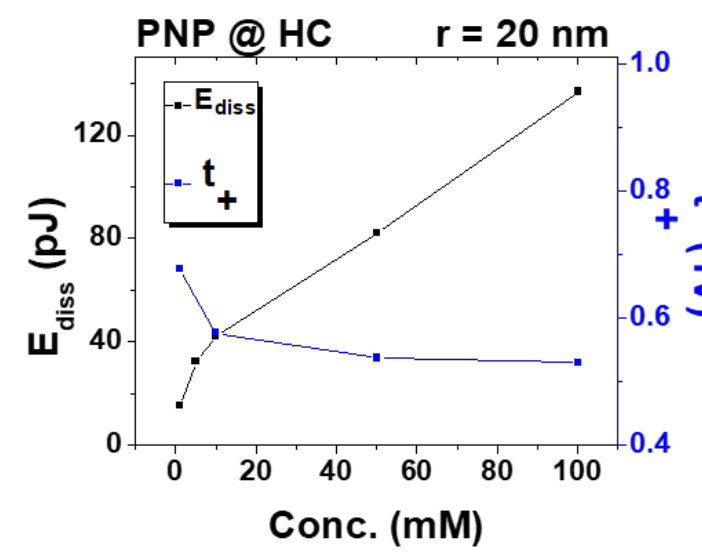

(B)

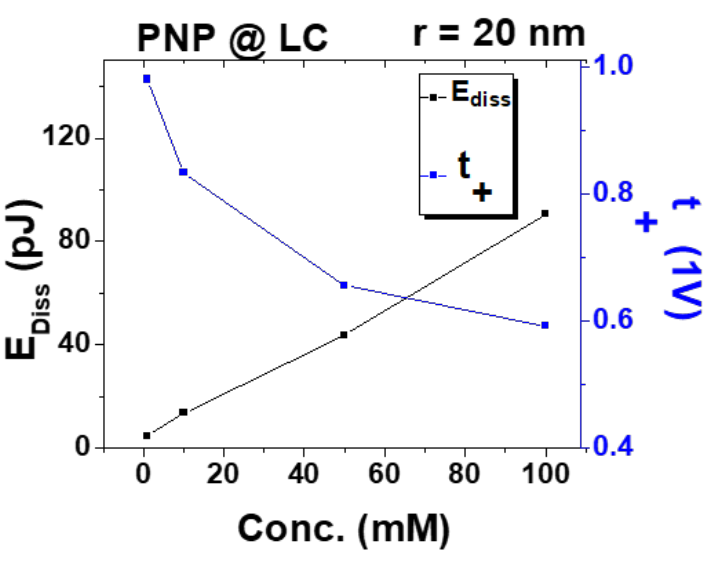

Figure S 12. The energy dissipation and cation selectivity from PNP model at (A) HC and (B) LC from a $20 \mathrm{~nm}$ nanopore. 
1. Liu, J.; Wang, D.; Kvetny, M.; Brown, W.; Li, Y.; Wang, G. Quantification of Steady-State Ion Transport through Single Conical Nanopores and a Nonuniform Distribution of Surface Charges. Langmuir 2013, $29,8743-8752$. 